Ostromisslensky: Neue empfindl. Farbenreaktion. 489

\begin{tabular}{ccc} 
Berechnet für & \multicolumn{2}{c}{ Gefunden: } \\
$\mathrm{C}_{7} \mathrm{H}_{7} \mathrm{O}:$ & I. & II. \\
$\mathbf{C}=78,50$ & 78,27 & $\mathbf{7 8 , 4 2} \%$ \\
$\mathrm{H}=6,54$ & 6,63 & $\mathbf{6 , 4 1} \%$.
\end{tabular}

Über die Bildungsweise und die Natur dieses Körpers von der empirischen Formel $\mathrm{C}_{7} \mathrm{H}_{7} \mathrm{O}$ konnten wir mangels genügender Mengen Substanz nichts Näheres ermitteln.

\title{
Über eine neue sehr empfindliche Farbenreaktion anf Äthylenbindungen, bzw. auf tautomere Formen; ${ }^{1}$ ) \\ Von \\ Iwan Ostromisslensky.
}

[Mitteilungen aus dem Chemischen Laboratorium der Kaiserl. Technischen Hochschule zu Moskau.]

Das Tetranitromethan ${ }^{2}$ ) ist eins der empfindlichsten Reagenzien auf Äthylenbindungen. Fügt man zu einer Lösung irgend einer Substanz ein paar Tropfen von diesem Reagens, so gibt die sofort eintretende meistens gelbe, orange, oder bräunliche Färbung den Beweis der Anwesenheit von Äthylenbindungen. Dieselbe Reaktion geht beim direkten Zusammenbringen der Komponente auch in festem Zustande vor sich. Die zu prüfende Lösung muß entweder neutral, oder sauer reagieren; schwach alkalische Lösungen (wie auch einige $A$ mine) zersetzen das Tetranitromethan unter Bildung der entsprechenden intensiv gelb gefärbten Nitroformsalze.

Das Dibromdinitromethan reagiert meistens mit ver-

1) Vergl. Ostromisslensky: Journ. russ. phys. chem. Ges. 12, Nr. 5; S.731-737; Werner, Ber. 42, 4324 (1909); Ostromigslensky, Ber. 43, 198 (1910); Arbeiten im Labor. für organ. Chem. der Moskauer Kaiserl. Techn. Hochschule, 6, S. 27-82 (1910); „Über die Natur der Äthylenbindungen", Moskau 1910, S. 30-74.

2) Über die Darstcllung dieser jetzt leicht zugänglichen Substanz aus Essigsäureanhydrid vergleiche: E. Berger, Compt. rend. 151, 813 (1910); F. D. Chattaw ay: Journ. Chem. Soc. 97, 2099 (1910) u. Chem. News 102, 307 (1910). 
490 Ostromisslensky: Nene empfindl. Farbenreaktion.

schiedenen Äthylenen ähnlich wie $\mathrm{C}\left(\mathrm{NO}_{2}\right)_{4}$, die entsprechenden Färbungen sind aber nicht so intensiv wie bei letzterem; noch weniger deutlich tritt die Reaktion mit Nitroform ein; beim Zusammenbringen der Äthylene mit aliphatischen Mononitroverbindungen $\left(\mathrm{CH}_{3} \mathrm{NO}_{2} ; \mathrm{C}_{2} \mathrm{H}_{5} \mathrm{NO}_{2}\right.$ usw.) entsteht - auch beim Erwärmen - keine Färbung.

I. Kohlenwasserstoffe. auftritt).

(Das Zeichen - bedeutet, daB mit $\mathrm{C}\left(\mathrm{NO}_{2}\right)_{4}$ keine Färbung

1. Methan -

2. Äthan -

4. Hexan -;

7. Methylhexamethylen -;

3. Butan -

5. Heptan -;

8. Pentamethylen -;

6. Cyclohexan -; 9. Gasolin -;

10. Ligroin -; 11. Petroläther -; 12. Paraffin -:

13. Vaselin -; 14. Petroleum -.

Alle diese Stoffe wurden zuvor von Äthylenderivaten auf üblichem Wege befreit. (Behandeln mit Brom oder $\mathrm{H}_{2} \mathrm{SO}_{4}$ usw.).

15. Äthylen: hellgelb.

21. Terpentinöl: rötlichgelb.

16. $A$ 1,3-Butadien: hellgelb.

22. Pinen: rötliehgelb.

17. Hexylen: gelb.

23. Camphen: bräunlichrot. ')

18. Amylen: rötliehgelb.

24. Menthen:

19. Dihydrobenzol: rötlichbraun.

25. Guajen: dunkelrot. ${ }^{2}$ )

20. Tetrahydrobenzol: rotbraun. 26. Dihydroguajen: rötlichbraun. ${ }^{3}$ )

Fügt man zu etwa $10 \mathrm{ccm}$ des Petroläthers (oder eines anderen Lösungsmittels der Grenzreihe) 2-5 Tropfen des Hexylens, Dihydrobenzols usw., so wird durch Zusatz des $\mathrm{C}\left(\mathrm{NO}_{2}\right)_{4}$ eine deutlich wahrnehmbare gelbe Färbung sofort hervorgerufen. Das Reagens stellt also eine der empfindlichsten Reaktionen

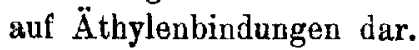

\section{Alkohole.}

27. Methylalkohol.- 29. Isobutylalkohol. - 31. Caprylalkobol. -

28. Äthylalkohol. - 30. Propylalkohol. - 32. Amylalkohol. -

33. Menthol. - 34. Cetylalkohol. - 35. Mannit. -

36. Glycerin. - 37. Glykol.

38. Allylalkohol: orangegelb.

39. Guajol: bräunlichrot.

40. Oleinalkohol: gelblichrot.
41. Isoeugenol: bräunlicbrot.

42. Eugenol:

43. Cholesterin: hellgelb.

1) In festen Aggregatzustande wird das Camphen durch $\mathrm{C}\left(\mathrm{NO}_{2}\right)_{4}$ intensiv goldgelb gefärbt.

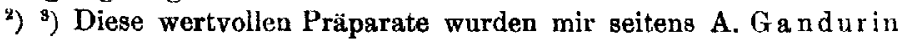
zur Verfügung gestellt. (Vergl. A. Gandurin: Ber. 42, 1809). 
III. Ketone.
44. Aceton -
48. Ionon: bräunlich.
45. Methyläthylketon -
49. Citral: orange.
46. Diäthylketon -
50. Eucarvon: blutrot.
47. Campher -

IV. Äther und Ester.

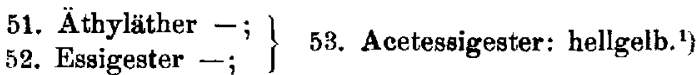

V. Aromatische Verbindungen.

54. Benzol: hellgelb.

э5. Toluol: ",

56. Xylole: "

57. Cumol: ",

58. Diphenyl: "

ð9. Chrysen: bräunlichrot.

60. Naphthalin: orangerot.

61. Anthracen: rosarot.

62. Reten: orange.

63. A cenaphthen : blutrot.

64. Pyren: dunkelrot.

65. Phenol: orangerot.

66. Brenzeatechin: orangerot. ${ }^{\text {) }}$ )

67. Resorcin: rot. $^{3}$ )

68. Hydrochinon: rötlichbraun. 4)
69. Pyragallol: braunrot.

70. Anisol: intensiv rot.

71. Phenetol:

72. Cumarin: kanariengelb.

73. Vanillin: orangegelb.

74. $\alpha$-Naphthol: braunrot.

75. $\beta$ -

76. Anilin: braunrot.

77. Toluidin: schwarz.

78. Monomethylanilin: dunkelbraun.

79. Dimethylanilin:

80. Diphenylcarbamid: hellgelb.

81. Naphthylamine $(\alpha$ u. $\beta)$ : braunrot.

82. Pyridin: hellgelb.

83. Chinolin: bräunlichrot.

84. Tannin: anfangs gelb, dann braun und schlieblich blutrot. ${ }^{5}$ )

\section{Nitroverbindungen}

geben mit $\mathrm{C}\left(\mathrm{NO}_{2}\right)_{4}$ gar keine Färbung. Untersucht wurden:

84. Mononitrobenzol -;

87. 1,3,5-Trinitrobenzol -;

85. o-, m-, p-Dinitrobenzol - ;

86. Trinitroanilin -;

88. $0-, \mathrm{m} \cdot, \mathrm{p}-\mathrm{Nitrophenol} \mathrm{-;}$

89. m-Nitroanilin -;

90. Pikrylchlorid -.

\section{Carbonsäuren}

geben nicht alle Färbungen mit $\mathrm{C}\left(\mathrm{NO}_{2}\right)_{4}$ :

91. Fumarsäure -; 94. 4-3,5-Dihydrophthalsänre: gelb.

92. Maleinsäure -; 95. $A-24-$

93. Benzoesäure -; 96. A-4-Tetrahydrophthalsäure: "

1) Vergl. S. 493 dieser Abhandlung.

$\left.{ }^{2}\right)^{3}$ ) $\left.{ }^{4}\right)$ Vergl. S. 493, Fubnote ${ }^{1}$ ).

5) Beim Stehen der zuerst erwärmten alkoholiachen Tanninlösungen mit $\mathrm{C}\left(\mathrm{NO}_{2}\right)_{4}$ fällt allmäblich ein gelatinöser, schön rot gefärbter Niederscblag aus. 
492 Ostromisslensky: Neue empfindl. Farbenreaktion.

97. Anthranileäure: orangerot.

99. Sylvinsäure: bräunlichrot.

98. o-Methylamidophenylorthotoluido- 100. Kolophonium: essigsäure: rosarot.

Die Nitroverbindungen, oft auch die Carbonsäuren, kommen hier also nicht in Betracht. Die Äthylenbindungen aller übrigen Klassen der organischen Verbindungen sind durch $\mathrm{C}\left(\mathrm{NO}_{2}\right)_{4}$ sehr scharf nachzuweisen. Die hohe Empfindlichkeit der Reaktion erlaubt, die Gegenwart der Äthylene auch in verschiedensten Gemischen, Naturverbindungen, oder in rohen technischen Produkten scharf festzustellen. So gelingt es z. B. die Gegenwart der Äthylene in allen Naphthadestillaten, im Urin des gesunden Menschen (es ist von vornherein ein wenig zu konzentrieren), oder im rohen 90 prozent. käuflichen Holzgeiste mit $\mathrm{C}\left(\mathrm{NO}_{2}\right)_{4}$ nachzuweisen. Im letzteren Falle ist das Zusammenstehen der Komponente bei normaler Temperatur innerhalb ca. 6 Stunden notwendig.

Das Tetranitromethan als ein Kriterium für tautomere Formen.

Nach allgemein angenommener Ansicht repräsentieren die tautomeren Verbindungen nur im festen Aggregatzustande eine bestimmte Form; im geschmolzenen, bzw. gelösten, oder gasförmigen dagegen stellen sie bekanntlich eine gewisse den äußeren Bedingungen entsprecheinde Gleichgewichtslage zwischen den beiden, bzw. mehreren tautomeren Formen dar. Dementsprechend müßte das Phloroglucin:<smiles>O=C1CC(=O)C(=O)C1</smiles><smiles>CC1CC(C(=O)O)CC1C(=O)O</smiles>

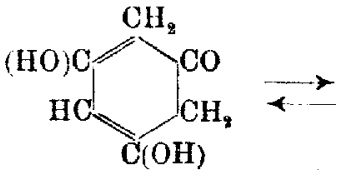<smiles>O=C[C@@H]1[CH][C@@H](C(=O)O)C(=O)C1</smiles>

1) Diese Strukturformel (des Hydroxydiketotetrahydrobenzols) schreiben Michael und $S m$ ith dem Phloroglncin im festen Aggregat. zustande zu. 
wie anch der Acetessigester:

$$
\mathrm{CH}_{3} \cdot \mathrm{CO} . \mathrm{CH}_{2} \cdot \mathrm{COOC}_{2} \mathrm{H}_{5} \longrightarrow \mathrm{CH}_{3} \cdot \mathrm{C}(\mathrm{OH}): \mathrm{CH} \cdot \mathrm{COOC}_{2} \mathrm{H}_{5} \text {, }
$$

sich durch $\mathrm{C}\left(\mathrm{NO}_{2}\right)_{4}$ als Derivate des Äthylens färben.

Das Experiment bestätigte diese Voraussetzung. Phloroglucin gibt mit $\mathrm{C}\left(\mathrm{NO}_{2}\right)_{4}$ in alkoholischen, bzw. wäBrigen oder ätherischen Lösungen eine intensive bräunlichrote Färbung; ${ }^{1}$ ) Acetessigester färbt sich mit demselben Reagens hell goldgelb. ${ }^{2}$ )

Die Zulässigkeit des $\mathrm{C}\left(\mathrm{NO}_{2}\right)_{4}$ als Reagens auf tautomere Formen habe ich durch Untersuchung eben derjenigen verhältnismäBig seltenen Verbindungen gezeigt, deren tautomere Formen vereinzelt isolierbar sind. Es ergab sich, daB nur die Enolform des Benzylidenbisacetessigesters $\left(\alpha^{\prime}\right):{ }^{3}$ )

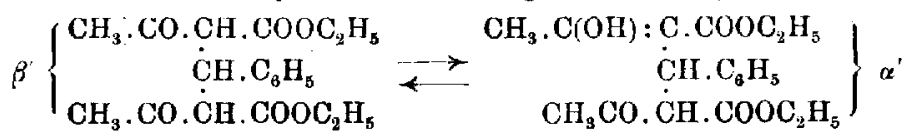

sich durch $\mathrm{C}\left(\mathrm{NO}_{2}\right)_{4}$, sowohl im festen Zustande, als auch in verschiedenen Lösungen (alkoholischen, ätherischen usw.) momentan zitronengelb färbt. Der $\beta_{1}$-Ester (also die Keto-Form) bleibt unter denselben Bedingungen vollständig farblos. Nur beim längeren Stehen der letzteren Lösung (ca. 2-5 Tage) wird allmählich die gelbe Färbung hervorgerufen, die mit derjenigen des $\alpha_{1}$-Esters identisch ist. Bei großem Überschuß an $\mathrm{C}\left(\mathrm{NO}_{2}\right)_{4}$ wird der $\beta_{1}$-Ester relativ schnell enolisiert (innerhalb 6 Stunden). Das Natriumsalz des Esters gibt mit $\mathrm{C}\left(\mathrm{NO}_{3}\right)_{4}$ behandelt eine intensive goldgelbe Färbung.

1) Es ergab sich zugleich, daB Phlorogluein und Dioxybenzole - im Gegensatze zu den übrigen $\ddot{A}$ thylenderivaten - mit $\mathrm{C}\left(\mathrm{NO}_{2}\right)_{4}$ im festen $\mathrm{Zu}$. stande anch beim Erwärmen nicht reagieren. Spuren von Wasser, bzw. Alkohol usw., rufen aber sofort eine intensive Färbung hervor. Diese Eigentümlichkeit der erwähnten aromatischen Pbenole ist offenbar durch ihre Unlöslichkeit in $\mathrm{C}\left(\mathrm{NO}_{2}\right)_{4}$ bedingt.

$\left.{ }^{2}\right) \mathrm{Es}$ wäre von Interesse, das Verbalten der beiden, gegenwärtig von $\mathrm{Kn}$ orr isolierten tautomeren Formen des Acetessigesters zu $\mathrm{C}\left(\mathrm{NO}_{2}\right)_{4}$ zu erforschen. (Vergl. L. Knorr, Ber. 44, 1138 [1911]).

s) Der Ketoester $\left(\beta_{1}\right)$ wurde nach Knövenagel durch Zusammen. bringen des Benzaldehyds mit Acetessigester in Gegenwart des Piperidins hergestellt. (Ann. Chem. 281, 29 [1894]). Den $\alpha_{1}$.Ester babe ich aus seinem Natriumsalz nach $R$ abe isoliert (Ann. Chem. 315, 176 [1900]). Die Reiuheit der beiden Verbindungen wurde durch mehrmalige Analyse (C und $\mathrm{H}$ ) geprüft. 
494 Ostromisslensky: Neue empfindl. Farbenreaktion.

Auch habe ich die tautomeren Formen des nach L. Knorr hergestellten Diacetbernsteinsäureesters:

$$
\begin{aligned}
& \mathrm{CH}_{3} \cdot \mathrm{C}(\mathrm{OH}): \mathrm{C} \cdot \mathrm{COOC}_{2} \mathrm{H}_{5} \longrightarrow \mathrm{CH}_{3} \cdot \mathrm{CO} \cdot \mathrm{CH} \cdot \mathrm{COOC}_{2} \mathrm{H}_{5} \\
& \mathrm{CH}_{3} \cdot \mathrm{C}(\mathrm{OH}): \mathrm{C} \cdot \mathrm{COOCO}_{2} \mathrm{H}_{5} \longrightarrow \mathrm{CH}_{3} \cdot \mathrm{CO} \cdot \mathrm{CH} \cdot \mathrm{COOO}_{2} \mathrm{H}_{5}
\end{aligned}
$$

in derselben Richtung untersucht.

Es ergab sich, daB hier die Enolisation der Ketoform bei Gegenwart von $\mathrm{C}\left(\mathrm{NO}_{2}\right)_{4}$ relativ schneller vor sich geht (innerhalb ca. 10-20 Minuten). Das Tetranitromethan stellt somit neben Eisenchlorid ein Reagens auf tautomere Formen dar.

\section{Das Tetranitromethan als Oxydationsmittel.}

Das Tetranitromethan ist - ähnlich dem Kupferchlorid, Chloranil usw. - ein schwaches Oxydationsmittel. Es oxydiert z. B. Hydrochinon zu Chinon und Dimethylanilin zu Krystall. violett.

Oxydation des Bydrochinons: Fügt man zu einer absolut alkoholischen Lösung des Hydrochinons einige Tropten des Tetranitromethans, so entsteht nur eine bräunliche Färbung, die offenbar dem Additionsprodukte angehört; sind aber in Lösung Spuren von Wasser anwesend, so tritt nach und nach die Temperaturerhöhung ein; es entwickelt sich ein anfangs farbloses Gas und aus der Lösung scheidet sich in dunkelgrün schimmernden Nädelchen ein krystallinischer Niederschlag aus. Der letztere wurde unter Druck abfiltriert und mit kaltem Alkohol rasch gewaschen. Die Analyse ergab:

$0,1790 \mathrm{~g}$ gaben $0,432 \mathrm{~g} \mathrm{CO}_{2}$ und $0,0734 \mathrm{~g} \mathrm{H}_{2} \mathrm{O}$.

$$
\begin{aligned}
& \mathrm{C}_{8} \mathrm{H}_{4} \mathrm{O}_{9} . \mathrm{C}_{6} \mathrm{H}_{4}(\mathrm{OH})_{9} \text {. Ber. C } 66,05 \text { H } 4,59 \% \\
& \text { Gef. C 65,82 H 4,56 " . }
\end{aligned}
$$

Die Substanz schmilzt bei $170^{\circ}$ und ist offenbar mit dem Chinhydron identisch. Das schon oben erwähnte, nicht brennbare, anfangs farblose Gas, das sich an der Luft rasch bräunt, erwies sich bei näherer Prüfung als NO (Reaktion mit Eisenvitriol); in der alkoholischen Mutterlauge konnte ich das Vorhandensein der salpetrigen Säure (Diazoreaktion), sowie der Salpetersäure (durch Nitron) feststellen. Während der Reaktion bildet sich kein Nitroform; es wurde versucht, dasselbe in Form seines schwerlöslichen sehr charakteristischen Kalium. salzes zu isolieren, aber ohne Erfolg. 
Ostromisslensky: Uber die Nitroalkylate. 495

Wegen der oxydierenden Wirkung der Nebenprodukte (Salpetersäure) ist der ganze Vorgang äußerst kompliziert.

Oxydation des Dimethylanilins: $Z$ u einer Lösung des Dimethylanilins in Schwefelkohlenstoff fügt man etwas $\mathrm{C}\left(\mathrm{NO}_{2}\right)_{4}$; es entsteht sofort eine dunkle fast schwarze Färbung. Man entfernt das Lösungsmittel durch langsames Verdunsten bei normaler Temperatur und läßt das Gefä $B$ mit übrigbleibender Masse längere Zeit (ca. 2-3 Tage) im Vakuum über $\mathrm{H}_{2} \mathrm{SO}_{4}$ stehen. Auf diese Weise erhält man bronzeglänzende, dunkelviolett schimmernde, harte Kryställchen, die sich in konzentrierter $\mathrm{HCl}$ mit bräunlicher Färbung auflösen; beim allmählichen Verdünnen dieser lösung mit Wasser wird sie anfangs grün und schlieblich blau gefärbt. Die Substanz zeigt alle für Krystallviolett charakteristische Reaktionen.

\section{Über die Nitroalkylate;}

von

\section{Iwan Ostromisslensky.}

Die Färbungen, die beim Zusammenbringen der aliphatischen Nitroverbindungen mit verschiedenen Äthylenderivaten entstehen, gehören offenbar den entsprechenden Additionsprodukten an, - also "einer neuen Klasse" organischer Verbindungen, die den sogen. „Pikraten“ u. dgl. vollkommen analog sind; diese Additionsprodukte sind als Zwischenstufe eines Oxydationsvorganges aufzufassen (vgl. oben).

Ich schlage vor, diese Verbindungen als "Nitroalkylate" zu bezeichnen; dann ist z. $\mathrm{B}$. die Verbindung des $\mathrm{C}\left(\mathrm{NO}_{2}\right)_{4}$ mit Acenaphthen als "Tetranitromethanat des Acenaphthens", diejenige des Bromdinitrobutans als „Bromdinitrobutanat" usw. zu bezeichnen.

Die Tetranitromethanate der aromatischen polycyclischen Kohlenwasserstoffe sind relativ die beständigsten. Sie können 